\title{
A NEW DEFINITION OF CONTINUOUS FRACTIONAL HARTLEY TRANSFORM
}

\author{
Soo-Chang Pei $i^{1} \quad$ Chien-Cheng Tseng ${ }^{2} \quad$ Min-Hung Yeh ${ }^{1} \quad$ Ding Jian-Jiun ${ }^{1}$ \\ ${ }^{1}$ Department of Electrical Engineering, National Taiwan University, Taipei, Taiwan, R. O. C. \\ Email address: pei@cc.ee.ntu.edu.tw \\ ${ }^{2}$ Department of Electronics Engineering, Hwa Hsia College of Technology and Commerce, Taipei, Taiwan, R.O.C.
}

\begin{abstract}
This paper is concerned with the definition of the continuous fractional Hartley transform. First, a general theory of linear fractional transform is presented to provide a systematic procedure to define the fractional version of any well-known linear transforms. Then, the results of general theory are used to derive the definitions of fractional Fourier transform (FRFT) and fractional Hartley transform (FRHT) which staisfiy the boundary conditions and additive property simultaneously. Next, an important relationship between FRFT and FRHT is described. Finally, a numerical example is illustrated to demonstrate the transform results of delta function of FRHT.
\end{abstract}

\section{INTRODUCTION}

In recent years, the concept of fractional operator and measure have been investigated extensively in many engineering applications and science. Four typical examples are described as follows. The first is fractional derivative and integral are defined by many mathematicians and applied to solve some physical problems [1]. The second is the fractional Fourier transform has been studied in the optic community and signal processing area [2]-[3]. The third is the fractional dimension is used to measure some real-world data such as coastline, clouds, dust in the air, and network of neurons in the body [4]. The fractional dimension has being applied widely to pattern recognition and classification. The last is fractional lower-order moment has been used to analyize non-Gaussian signal which is more realistic than the Gaussian model in signal processing applications [5].

On the other hand, various unitary transforms have been widely used in image compression and adaptive filtering except Fourier transform. Some typical ones are cosine transform, sine transform, and Hartley transform e.t.c. So far, the fractional version of these transforms have been generalized [6]. However, the fractional Hartley transform defined in [6] does not obey additivity property which means that application of the transform with angular parameter $\alpha$ followed by an application of the transform with angular parameter $\beta$ is equivalent to the application of the transform with angular parameter $\alpha+\beta$. The purpose of this paper is to present a new fractional Hartley transform which obeys additivity property exactly.

\section{LINEAR FRACTIONAL TRANSFORM}

Let $T$ be a linear transform that maps function $f(x)$ into the function $g(u)$, i.e.,

$$
T(f(x))=g(u)
$$

where variables $x, u$ are different because the domain would change after transformation. Then, the corresponding fractional transform of $T$ is defined by

$$
T^{\alpha}(f(x))=g^{\alpha}(u)
$$

which must satisfy the boundary conditions

$$
\begin{aligned}
& T^{0}(f(x))=f(x) \\
& T^{1}(f(x))=g(u)
\end{aligned}
$$

and the additive property

$$
T^{\beta}\left(T^{\alpha}(f(x))=T^{\alpha}\left(T^{\beta}(f(x))=T^{\alpha+\beta}(f(x))\right.\right.
$$

Given the transform $T$, the problem is how to define $T^{\alpha}$ such that boundary condition and additive property are satisfied. A systematic procedure will be described as follows: Let $e_{n}(x)$ be an eigenfunction of linear transform $T$ with eigenvalue $\lambda_{n}$, then we have

$$
T\left(e_{n}(x)\right)=\lambda_{n} e_{n}(u)
$$

If $e_{0}(x), e_{1}(x), \cdots, e_{\infty}(x)$ is an orthonormal basis in the $x$ domain, the function $f(x)$ can be rewritten as

$$
f(x)=\sum_{n=0}^{\infty} a_{n} e_{n}(x)
$$

where the coefficient $a_{n}$ is given by

$$
a_{n}=<f(x), e_{n}(x)>=\int_{-\infty}^{\infty} f(x) e_{n}(x) d x
$$

The notation $<<_{j}>$ denotes the inner product. Then, the transform of $f(x)$ is given by

$$
T(f(x))=T\left(\sum_{n=0}^{\infty} a_{n} e_{n}(x)\right)=\sum_{n=0}^{\infty} a_{n} \lambda_{n} e_{n}(u)
$$

Based on this equation, the fractional transfom $T^{\alpha}$ is defined as follows:

$$
T^{\alpha}(f(x))=\sum_{n=0}^{\infty} a_{n} \lambda_{n}^{\alpha} e_{n}(u)
$$

Substitute eq(6) into eq(8), we obtain

$$
\begin{aligned}
T^{\alpha}(f(x)) & =\sum_{n=0}^{\infty} a_{n} \lambda_{n}^{\alpha} e_{n}(u) \\
& =\sum_{n=0}^{\infty}\left[\int_{-\infty}^{\infty} f(x) e_{n}(x) d x\right] \lambda_{n}^{\alpha} e_{n}(u) \\
& =\int_{-\infty}^{\infty} K^{\alpha}(x, u) f(x) d x
\end{aligned}
$$


where transform kernel is defined by

$$
K^{\alpha}(x, u)=\sum_{n=0}^{\infty} \lambda_{n}^{\alpha} e_{n}(x) e_{n}(u)
$$

From eq(9), it is clear that $T^{\alpha}$ is a linear transform. And, from eq(5)(7)(8), it can be shown that the boundary conditions are satisfied. Since the expression

$$
T^{\alpha}\left(e_{n}(x)\right)=\lambda_{n}^{\alpha} e_{n}(u)
$$

holds and $T^{\alpha}$ is linear, we have

$$
\begin{aligned}
T^{\alpha}\left(T^{\beta}(f(x))\right) & =T^{\alpha}\left(\sum_{n=0}^{\infty} a_{n} \lambda_{n}^{\beta} e_{n}(u)\right) \\
& =\sum_{n=0}^{\infty} a_{n} \lambda_{n}^{\beta}\left[T^{\alpha}\left(e_{n}(u)\right)\right] \\
& =\sum_{n=0}^{\infty} a_{n} \lambda_{n}^{\beta}\left[\lambda_{n}^{\alpha} e_{n}(u)\right] \\
& =\sum_{n=0}^{\infty} a_{n} \lambda_{n}^{\alpha+\beta} e_{n}(u)=T^{\alpha+\beta}(f(x))
\end{aligned}
$$

This tells us that the additive ptoperty is also satisfied. Given linear transform $T$, the procedure to find its fractional transfrom is now summarized as follows:

Step 1: Find the eigenfunctions $e_{n}(x)$ and eigenvalues $\lambda_{n}$ of transform $T$ for $n=0,1, \cdots \infty$.

Step 2: Use eq(10) to compute the transform kernel $K^{\alpha}(x, u)$. The closed-form formula may be obtained.

Step 3: The fractional transform of $f(x)$ is then given by

$$
T^{\alpha}(f(x))=\int_{-\infty}^{\infty} K^{\alpha}(x, u) f(x) d x
$$

In next two subsections, we will use this procedure to derive the fractional transforms of Fourier transform and Hartley transform. Finally, it is worth mentioning that if there exists a minimum positive number $p$ such that

$$
\lambda_{n}^{p}=1 \quad \text { for } n=0,1, \cdots, \infty
$$

holds, then substituting eq(12)(5) into eq(8) yields

$$
T^{p}(f(x))=f(u)
$$

Thus, the $p$ is called the period of fractional transform $T^{\alpha}$.

\section{FRACTIONAL FOURIER TRANSFORM}

If linear transform $T$ is well-known Fourier transform, then it is defined by

$$
T(f(x))=g_{F}(u)=\frac{1}{\sqrt{2 \pi}} \int_{-\infty}^{\infty} f(x) e^{-j x u} d x
$$

The eigenfunctions $e_{n}(x)$ and eigenvalues $\lambda_{n}$ of Fourier transform are given by

$$
\begin{aligned}
e_{n}(x) & =\sqrt{\frac{1}{2^{n} n ! \sqrt{\pi}}} e^{\frac{-x^{2}}{2}} H_{n}(x) \\
\lambda_{n} & =e^{-j n \frac{\pi}{2}}
\end{aligned}
$$

where $H_{n}(x)$ is the $n$th order Hermite polynomial. Thus, the transform kernel $K_{F}^{\alpha}(x, u)$ of fractional Fourier transform can be calculated as follows:

$$
\begin{aligned}
K_{F}^{\alpha}(x, u) & =\sum_{n=0}^{\infty} \lambda_{n}^{\alpha} e_{n}(x) e_{n}(u) \\
& =\left[\sum_{n=0}^{\infty} \frac{e^{-j n \frac{\alpha \pi}{2}}}{2^{n} n ! \sqrt{\pi}} H_{n}(x) H_{n}(u)\right] e^{\frac{-\left(x^{2}+u^{2}\right)}{2}(16)}
\end{aligned}
$$

In order to obtain the closed-form representation, we use the following formula:

$$
\begin{aligned}
& \sum_{n=0}^{\infty} \frac{e^{-j n \frac{\alpha \pi}{2}}}{2^{n} n ! \sqrt{\pi}} H_{n}(x) H_{n}(u) \\
= & \frac{1}{\sqrt{\pi} \sqrt{1-e^{-j \alpha \pi}}} \exp \left[\frac{2 x u e^{-j \frac{\alpha \pi}{2}}-e^{-j \alpha \pi}\left(x^{2}+u^{2}\right)}{1-e^{-j \alpha \pi}}\right]
\end{aligned}
$$

Substitute eq(17) into eq(16) and using the following equalities:

$$
\begin{aligned}
& \frac{1}{\sqrt{\pi} \sqrt{1-e^{-j \alpha \pi}}}=\sqrt{\frac{1-j \cot \phi}{2 \pi}} \\
& \frac{2 x u e^{-j \frac{\alpha \pi}{2}}}{1-e^{-j \alpha \pi}}=-j x u \csc \phi \\
& \frac{-e^{-j \alpha \pi}\left(x^{2}+u^{2}\right)}{1-e^{-j \alpha \pi}}-\frac{\left(x^{2}+u^{2}\right)}{2}=j \frac{x^{2}+u^{2}}{2} \cot \phi
\end{aligned}
$$

where $\phi=\frac{\alpha \pi}{2}$, we have

$$
K_{F}^{\alpha}(x, u)=\sqrt{\frac{1-j \cot \phi}{2 \pi}} e^{j \frac{x^{2}+u^{2}}{2} \cot \phi-j x u \csc \phi}
$$

This kernel has been widely used in the signal processing community [3] and fractional Fourier transform of function $f(x)$ is defined by

$$
g_{F}^{\alpha}(u)=\int_{-\infty}^{\infty} K_{F}^{\alpha}(x, u) f(x) d x
$$

Based on this equation, it can be shown that if $f(x)$ is an even function, then its fractional Fourier transform $g_{F}^{\alpha}(u)$ is an even function. From eq(15), we have

$$
\lambda_{n}^{4}=e^{-j 2 n \pi}=1 \quad \text { for } n=0,1, \cdots, \infty
$$

Thus, the period of fractional Fourier transform is 4 .

\section{FRACTIONAL HARTLEY TRANSFORM}

If linear transform $T$ is well-known Hartley transform, then it is defined by

$$
T(f(x))=g_{H}(u)=\frac{1}{\sqrt{2 \pi}} \int_{-\infty}^{\infty} f(x) \operatorname{cas}(x u) d x
$$

where $\operatorname{cas}(x u)=\cos (u x)+\sin (u x)$. From the eq(14)(21), the relation between Fourier transform and Hartley transform is given by

$$
g_{H}(u)=\frac{1+j}{2} g_{F}(u)+\frac{1-j}{2} g_{F}(-u)
$$


From the eq(15)(22) and the symmetric property of Hermite polynominal

$$
H_{n}(-x)= \begin{cases}H_{n}(x) & \text { if } n \text { is even } \\ -H_{n}(x) & \text { if } n \text { is odd }\end{cases}
$$

the eigenvectors of Hartley transform are same as those of Fourier transform, but the eigenvalues $\lambda_{n}$ of eigenvector $e_{n}(x)$ are different and given by

$$
\lambda_{n}= \begin{cases}e^{-j n \frac{\pi}{2}} & \text { if } n \text { is even } \\ e^{-j(n-1) \frac{\pi}{2}} & \text { if } n \text { is odd }\end{cases}
$$

Thus, the transform kernel $K_{H}^{\alpha}(x, u)$ of fractional Hartley transform can be calculated as follows:

$$
\begin{aligned}
K_{H}^{\alpha}(x, u) & =\sum_{n=0}^{\infty} \lambda_{n}^{\alpha} e_{n}(x) e_{n}(u) \\
& =[E(x, u)+O(x, u)] e^{\frac{-\left(x^{2}+u^{2}\right)}{2}}
\end{aligned}
$$

where $E(x, u)$ and $O(x, u)$ are defined by

$$
\begin{aligned}
& E(x, u)=\sum_{n=0}^{\infty} \frac{e^{-j n \alpha \pi}}{2^{2 n}(2 n) ! \sqrt{\pi}} H_{2 n}(x) H_{2 n}(u) \\
& O(x, u)=\sum_{n=0}^{\infty} \frac{e^{-j n \alpha \pi}}{2^{2 n+1}(2 n+1) ! \sqrt{\pi}} H_{2 n+1}(x) H_{2 n+1}(u)
\end{aligned}
$$

From eq(26) and (16), the fractional Fourier transform kernel $K_{F}^{\alpha}(x, u)$ can be rewritten as:

$$
K_{F}^{\alpha}(x, u)=\left[E(x, u)+e^{-j \frac{\alpha \pi}{2}} O(x, u)\right] e^{\frac{-\left(x^{2}+u^{2}\right)}{2}}
$$

Using the symmetric property of Hermite polynomial in eq(23), it can be shown that

$$
\begin{aligned}
& E(x,-u)=E(x, u) \\
& O(x,-u)=-O(x, u)
\end{aligned}
$$

From eq $(25)(27)(28)$, the relation between fractional Fourier transform kernel and fractional Hartley transform kernel is given by

$$
K_{H}^{\alpha}(x, u)=\frac{1+e^{j \frac{\alpha \pi}{2}}}{2} K_{F}^{\alpha}(x, u)+\frac{1-e^{j \frac{\alpha \pi}{2}}}{2} K_{F}^{\alpha}(x,-u)
$$

Substitute eq(18) into (29), the closed form formula of fractional Hartley transform kernel is

$$
\begin{array}{r}
K_{H}^{\alpha}(x, u)=\sqrt{\frac{1-j \cot \phi}{2 \pi}} e^{j \frac{x^{2}+u^{2}}{2} \cot \phi}(\cos (x u \csc \phi) \\
\left.+e^{j\left(\phi-\frac{\pi}{2}\right)} \sin (x u \csc \phi)\right)
\end{array}
$$

Based on this kernel, the fractional Hartley transform of function $f(x)$ is defined by

$$
g_{H}^{\alpha}(u)=\int_{-\infty}^{\infty} K_{H}^{\alpha}(x, u) f(x) d x
$$

It is clear that the fractional Hartley tansform $g_{H}^{\alpha}(u)$ of real function $f(x)$ is complex valued except $\phi=\frac{k \pi}{2}$ ( $k$ is odd integer). From eq(24), we have

$$
\lambda_{n}^{2}=1 \quad \text { for } n=0,1, \cdots, \infty
$$

Thus, the period of fractional Hartley transform is 2 . Moreover, from eq(29), it is easy to show that

$$
g_{H}^{\alpha}(u)=\frac{1+e^{j \frac{\alpha \pi}{2}}}{2} g_{F}^{\alpha}(u)+\frac{1-e^{j \frac{\alpha \pi}{2}}}{2} g_{F}^{\alpha}(-u)
$$

This is relation between fractional Fourier transform and fractional Hartley transform. When $\alpha=1$, the eq(33) becomes the eq(22) which is relation between Fourier transform and Hartley transform. Using eq(33), the fractional Hartley transform can be found from the fractional Fourier transform. For example, the fractional Fourier transform of delta function $\delta(x)$ is

$$
g_{F}^{\alpha}(u)=\sqrt{\frac{1-j \cot \phi}{2 \pi}} e^{j \frac{u^{2}}{2} \cot \phi}
$$

, so its fractional Hartley transform is given by

$$
g_{H}^{\alpha}(u)=\sqrt{\frac{1-j \cot \phi}{2 \pi}} e^{j \frac{u^{2}}{2} \cot \phi}
$$

Fig.1 shows this transform results $g_{H}^{\alpha}(u)$ for various $\phi=$ $\frac{\alpha \pi}{2}$. The real parts in this paper are plotted by solid lines, and imaginary parts are plotted by dashed or dotted lines. Finally, it is worth mentioning that if fractional Fourier transform $g_{F}^{\alpha}(u)$ is even function, then it can be shown that

$$
g_{H}^{\alpha}(u)=g_{F}^{\alpha}(u)
$$

, i.e., fractional Hartley transform is equal to fractional Fourier transform.

\section{CONCLUSIONS}

A new definition of the continuous fractional Hartley transform has been described. A general theory of linear fractional transform is also presented to provide a systematic procedure to define the fractional version of any well-known linear transforms. The proposed fractional Fourier transfor$m$ (FRFT) and fractional Hartley transform (FRHT) staisfiy the boundary conditions and additive property exactly. A numerical example is illustrated to demonstrate the transform results of delta function of FRHT.

\section{REFERENCES}

[1] K.S. Miller and B. Ross, An Introduction to the Fractional Calculus and Fractional Differential Equations, John Wiely, 1993.

[2] M.A. Kutay, H.M. Ozaktas, O. Arikan and L. Onural, " Optimal filtering in fractional Fourier domains", IEEE Trans. Signal Processing, vol. 45, pp.1129-1143, May 1997.

[3] S.C. Pei and C.C. Tseng, "A new discrete fractional Fourier transform based on constrained eigendecomposition of DFT matrix by Largrange multiplier method", in Proc. IEEE Int. Conf. Acoust. Speech, Signal Processing, pp.3965-3968, Munich German, Apr. 1997

[4] M.F. Barnsley, Fractal Everywhere, Boston:Academic, 1988.

[5] M. Shao and C.L. Nikias, "Signal processing with fractional lower order moment: stable processes and their applications", Proc. IEEE, vol. 81, pp. 986-1010, July, 1993.

[6] A.W. Lohmann, D. Mendlovic, Z. Zalevsky and R.G. Dorsch, "Some important fractional transformations for signal processing", Optics Communications, pp. 18-20, Apr. 1996. 

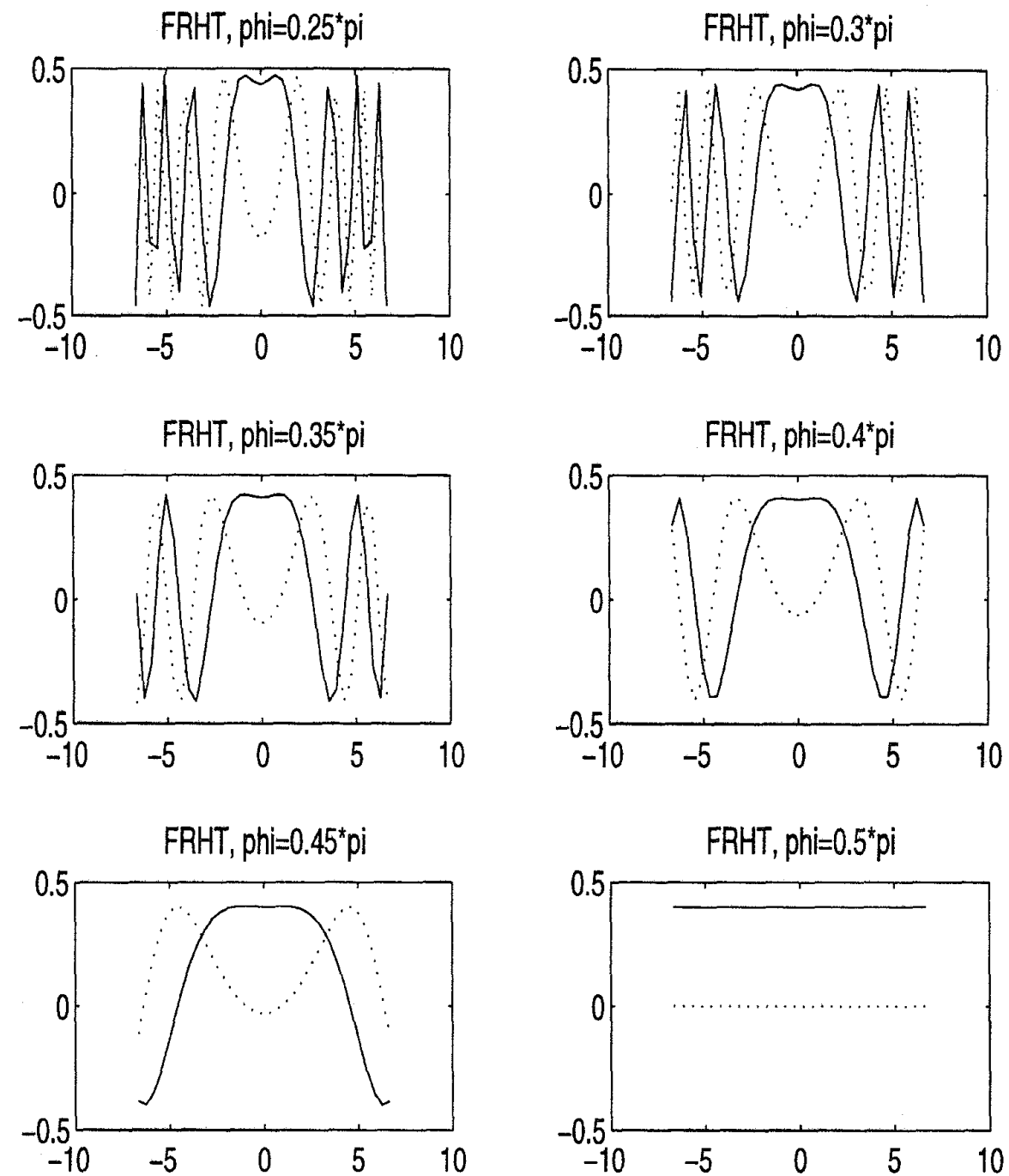

Fig. 1 The results of continuous fractional Hartley transform of the delta function $\delta(x)$ for various $\phi=\frac{\alpha \pi}{2}$. 